\title{
Aplicación del programa para mejorar la autonomía de los niños en etapa pre escolar
}

\section{Application of the program to improve the autonomy of pre-school children}

\author{
Angela María Teresa Samamé Gonzáles, ${ }^{1}$ Petronila Melchora Tasayco Pacheco ${ }^{2}$ \\ Juan Méndez Vergaray ${ }^{3}$ \\ Universidad César Vallejo, Lima, Peru ${ }^{123}$ \\ ORCID: https://orcid.org/0000-0001-1481-1862 1 \\ ORCID: https://orcid.org/0000-0002-2351-54912 \\ ORCID: https://orcid.org/0000-0001-7286-0534
}

Recibido: 01 de setiembre de 2020

Aceptado: 05 de enero de 2021

\section{Resumen:}

El objetivo de la investigación fue demostrar cómo la "Aplicación del Programa para mejorar la autonomía de los niños en etapa pre escolar" influye en la autonomía de los niños de 24 meses en un Pronoei de San Juan de Miraflores (Lima, Perú). El enfoque de investigación fue cuantitativo, de tipo aplicada o práctica, el diseño fue cuasi experimental, porque se utilizaron dos grupos y el método correspondió al hipotético deductivo. La población de estudio fueron 64 estudiantes. El instrumento fue una lista de chequeo que fue estructurada en tres dimensiones como fueron seguridad afectiva, libertad de movimiento, espacios y materiales apropiados. El programa tuvo una duración de doce semanas, para lo cual participaron activamente los padres de familia antes, durante y después de cada sesión de clases. Los resultados evidenciaron que el programa influyó positivamente en la autonomía de los niños, el cual fue demostrado con la aplicación de la prueba de U Mann Whitney, porque el nivel de significancia calculado en el pre test fue de 0.052 , y en el post test se registró .000, situación similar aconteció en las dimensiones. Finalmente, se concluye que la aplicación del programa mejora y refuerza la autonomía de los niños, razón por la cual deberá ser replicado en otros grupos.

Palabras clave: Autonomía, seguridad, afecto, movimiento, niños, educación, preescolar.

\footnotetext{
Abstract:

${ }^{1}$ Correspondencia al autor

E-mail: tere_samame@hotmail.com
}

The objective of the research carried out was to demonstrate that the application of the "Application of the program to improve the autonomy of pre-school children" Program influenced the autonomy of 24-month-old children in a Pronoei in the San Juan de Miraflores district (Lima, Peru). The research approach was quantitative, applied or practical, the design 
was quasi-experimental and the method corresponded to the hypothetical deductive one. The study population was 64 students. The instrument was a checklist that was structured in three dimensions, such as affective security, freedom of movement, appropriate spaces and materials. The program lasted twelve weeks, for which the parents actively participated before, during and after each class session. The results showed that the program had a positive influence on the autonomy of the children, which was demonstrated with the application of the U Mann Whitney test, because the level of significance calculated in the pre-test was 0.052 , and in the post-test it was registered .000 , a similar situation occurred in the dimensions. Finally, it is concluded that the application of the program improves and reinforces the autonomy of children, which is why it should be replicated in other groups.

Keywords: Autonomy, security, affection, movement, children, education, preschool.

\section{Introducción}

El desarrollo de la autonomía es una tarea importante que recae en los padres de familia, el excesivo control en el desenvolvimiento, la sobreprotección, el autoritarismo y otras situaciones afectan a la formación de los futuros ciudadanos (Blanco, Umayahara y Reveco, 2004). Por ejemplo, en Escocia existen las denominadas escuelas forestales, cuyo objetivo es el desenvolvimiento natural de los estudiantes, están rodeados de flora, fauna y humanos (Barrable, 2020). En Nicaragua se dejó en evidencia que la autonomía depende básicamente de la madre, aquellas que tienen un bajo nivel optan por sobreproteger al menor y amamantarlos por tiempo prolongado, mientras que quienes poseen un nivel medio, optan por desarrollar también la autonomía e implementan la alimentación complementaria a la lecha materna (Ziaei, y otros, 2015).

Cuervo (2010) expuso que la práctica de estilos de crianza agresivo, permisivos, autoritarios y negligentes afectan a la autonomía de los niños y el desempeño del futuro ciudadano (Oliveira, 2019). El Estado como ente rector incluye en el plan curricular el desempeño del niño, siendo un tema importante la autonomía y se desarrolla desde edades tempranas (Oliveira, 2019), sin embargo, para lograr los objetivos institucionales se requiere el involucramiento de los maestros y que tengan las competencias necesarias para el nivel inicial.

El Perú suscribió la Convención en relación a los derechos de los niños, por ello se promulgó el Código de los niños y adolescentes, además de diferentes planes de acción escolarizados como los servicios de cuna y cuna jardín; y no escolarizados como aprendiendo en el hogar, las ludotecas, las salas de educación temprana, familias que aprenden, entre otros; cuyo objetivo es el desarrollo psicomotor y cognitivo del infante, el mismo que debe estar acorde a la edad cronológica (Ministerio de la mujer y Poblaciones Vulnerables, 2012)

La educación inicial es una etapa muy importante en la formación y desarrollo del infante, sin embargo, existen padres que asumen actitudes sobre proteccionistas o conductas 
autoritarias, haciendo que los menores carezcan de iniciativa, capacidad para ingerir sus alimentos solos, sean sumisos, desorganizados, entre otros aspectos que afectan a su desenvolvimiento (Arciniegas, y otros, 2018). Por otra parte, el niño que juega, logra mejorar sus relaciones humanas, aprende a cuidarse, escuchar, expresarse con libertad y evita los peligros (Ministerio de Educación, 2013). En consecuencia, el desarrollo de la autonomía se logra a través de los hábitos, las interacciones sociales, el desarrollo intelectual, las actividades de ocio y responsabilidad (Gómez y Martin, 2013). Estudios previos han expuesto que los niños lograron la autonomía cuando se desenvuelven solos en sus actividades cotidianas como el colegio (Ayllón, et al., 2019), además el juego es un elemento importante y mejor si se desarrolla en los espacios públicos (Borgogni, 2019). El modelo actual de crianza liberal sostenida en dos pilares como es el respeto y la confianza hace que el infante se desenvuelva con libertad, desarrollando así la autonomía (Martakis, Brand, y Schroder-Back, 2018). Es importante para la educación de los niños la aplicación de juegos, nuevos hábitos, desarrollo de actividades de interacción social en lugares libres como los parques, la playa, espacios deportivos, entre otros, con el fin que el menor se desenvuelva libremente, y que ante diferentes circunstancias aprenda afrontar dicha situación, y evidencie el desarrollo de la autonomía.

En este sentido, Arizaca (2010) demostró que la sobreprotección se relaciona negativamente con la autonomía, recomendando la implementación de programas de intervención; Condori y Luque (2019) lograron mejorar la autonomía de los niños en actividades como el lavado de manos, dientes, baño, respeto, responsabilidad, y seguridad. Asimismo, el ambiente es necesario para el descubrimiento y el desarrollo de la autonomía (Yábar y Bronzoni, 2018). Peñaranda (2018) evidenció que solo el 40\% de docentes permiten que el niño participe libremente en el aula, el $80 \%$ no preguntan a los infantes sobre sus necesidades, menos desarrollan la autonomía, esto a razón que no existe armonía entre las competencias previstas y el desarrollo de las actividades.

La autonomía se sustenta en la teoría Psicogenética de Piaget, porque el niño a razón de la edad cronológica adquiere nuevos conocimientos, tiene facilidad para interrelacionarse con el entorno y controlar sus comportamientos (Arias-Arroyo, Merino-Zurita y Peralvo-Arequipa, 2017). La teoría sociocultural de Vigotsky (1978), sostuvo que el desarrollo del infante está vinculado a su entorno. Si el menor se desenvuelve en un ambiente armónico, con padres que otorgan responsabilidades y corrigen los comportamientos inadecuados, estará formando una persona autónoma, caso contrario ante el desfase ameritará la implementación de programas para mejorar el desarrollo psicológico del menor (Chaves, 2001; De Rosa, 2018).También se consideró la teoría del aprendizaje significativo de Ausbel, porque el niño cada día adquiere 
nuevos conocimientos, los mismos que se suman a los conocimientos previos, sin embargo otorgará significado cuando lo practique o utilice en su vida cotidiana (Baca, 2018; Carrera y Mazzarella, 2001). Asimismo, la teoría del aprendizaje por descubrimiento de Brunner es importante porque los menores adquieren el conocimiento a través de la curiosidad, el cual es motivado por el docente, porque en las explicaciones deja vacíos, que deberán ser absueltos por los niños (Arias, 2015).

Por otro lado, la autonomía es entendida como la capacidad que tiene el niño para realizar sus actividades por sí mismo de manera libre e independiente, para ello repite comportamientos que otorgaron éxito o logro (Morrison, 2005; Posada, Gómez y Ramírez, 2005; Salazar, 2017). En este caso, el comportamiento de los infantes en los primeros años de vida no es autónomo, responde a sus impulsos y reacciones, lo que conlleva a mirar, oler, morder, tocar, teniendo como respuesta una sonrisa, renegar, llorar, reír (Posada, Gómez y Ramírez, 2005), por lo tanto, el adulto cuidador tiene dos alternativas, la primera que es reforzar dicho comportamiento o por el contrario asumir con responsabilidad y corregir dichos actos. El estudio realizado fue importante porque los niños de 24 meses lograron desarrollar la autonomía, razón por la cual se aplicó un programa que tuvo una duración de 12 semanas. El objetivo fue demostrar que la aplicación del programa mejoró la autonomía de los niños en etapa pre escolar

\section{Metodología}

La investigación se realizó sustentado en el paradigma positivista, en vista que el interés del estudio radicó en la explicación y control, de tal forma que se logre generalizar los resultados obtenidos (Baena 2017; Hernández, Fernández, y Baptista 2014). El tipo de estudio fue práctico o de utilidad, el método correspondió al hipotético deductivo, el diseño fue cuasi experimental (grupo experimental y control), integrado por dos grupos equitativos de 32 estudiantes.

El programa de intervención se realizó por el espacio de doce semanas, en el mismo se desarrollaron talleres grupales e individuales dos veces por semana, donde a través del juego físico, funcional, constructivo y reglas con el uso del WhatsApp y Zoom se logró progresivamente desarrollar la autonomía, la medición se realizó con la lista de cotejo, la cual fue aplicada al inicio y final del programa.

La población de estudio estuvo conformada por 64 estudiantes, el criterio de inclusión correspondió a registrar matrícula en el año 2020, la edad estuvo comprendida entre 2 años y 2 años y 11 meses, además se obtuvo la autorización de los padres de familia. La técnica de 
recopilación de datos fue la evaluación y el instrumento la ficha de observación o cotejo, la misma que fue evaluada por tres dimensiones: a) Seguridad afectiva, b) Libertad de movimiento; c) Espacios y materiales apropiados, con un total de 20 ítems, la escala de calificación fue 0 incorrecto o no y 1 correcto o sí.

El instrumento se sometió a la validez del contenido con cinco expertos, quienes evaluaron que cada ítem responda a la dimensión, y que las dimensiones permitan medir la variable autonomía. La evaluación se realizó a través de los criterios de pertinencia, relevancia y claridad, donde cada ítem obtuvo la calificación de aplicable. Asimismo, se empleó la prueba de V Aiken, donde el valor calculado por todos los expertos fue de 1 o $100 \%$ de aprobación (Martin-Romera \& Molina, 2017). Además, el instrumento se sometió a la prueba de KR20 porque la escala fue dicotómica (correcto e incorrecto), la muestra estuvo integrada por 15 niños que no fueron parte del estudio, el valor calculado correspondió a .9954, lo cual indica que el instrumento tiene alta confiabilidad (Hernández, Fernández, \& Baptista, 2014), razón por la cual se aplicó a la muestra de estudio.

Los datos fueron analizados con la prueba de U Mann Whitney, porque los datos no presentaban distribución normal, por lo tanto el objetivo fue comparar las medianas entre los puntajes de ambos grupos (Rivas-Ruiz, Moreno-Palacios y Talavera, 2013; Rivas-Ruiz, Moreno-Palacios, y Talavera 2013; Rivas-Ruiz, Moreno-Palacios, y Talavera 2013; RivasRuiz, Moreno-Palacios, y Talavera 2013; Rivas-Ruiz, Moreno-Palacios, y Talavera 2013.

\section{Resultados}

Los resultados evidencian que los puntajes de la autonomía, seguridad afectiva, libertad de movimiento, espacios y materiales apropiados se vieron incrementados en el grupo experimental respecto al grupo control. El programa se realizó de forma virtual a cargo de la docente responsable y el personal de apoyo por la coyuntura que se vive en el presente año, además fue importante el compromiso y soporte de los padres de familia; a diferencia que el grupo control ha seguido las sesiones de clase del programa "Aprendo en casa".

En la tabla 1 se expone que los puntajes de la autonomía en el grupo experimental incrementaron 8.25 puntos en promedio (Pre=8.69; Post=16.94), mientras que en el grupo control disminuyó 0.28 puntos (Pre=9.75; Post=9.47). Respecto a la dimensión seguridad afectiva el grupo experimental incrementó 2.63 puntos en promedio (Pre=2.53; Post=5.16), mientras que en el grupo control disminuyó 0.25 puntos (Pre=2.75; Post=2.50); en relación a la dimensión libertad de movimiento, el grupo experimental incrementó 2.81 puntos en promedio (Pre=3.69; Post=6.50), mientras que en el grupo control disminuyó 0.19 puntos (Pre=4.06; 
Post=4.25); finalmente respecto a la dimensión libertad de movimiento, el grupo experimental incrementó 2.81 puntos en promedio ( $\mathrm{Pre}=2.47$; Post=5.28), mientras que en el grupo control disminuyó 0.19 puntos (Pre=2.94; Post=2.72).

\section{Tabla 1}

Estadísticos descriptivos de la autonomía y dimensiones

\begin{tabular}{|c|c|c|c|c|c|c|c|}
\hline & Grupo & Prueba & $\mathrm{N}$ & Mínimo & Máximo & Media & Desviación \\
\hline \multirow[t]{4}{*}{ Vd: Autonomía } & Experimental & Pre test & 32 & 5 & 12 & 8,69 & 1,925 \\
\hline & & Pos test & 32 & 13 & 20 & 16,94 & 1,501 \\
\hline & Control & Pre test & 32 & 5 & 14 & 9,75 & 2,185 \\
\hline & & Pos test & 32 & 3 & 16 & 9,47 & 2,951 \\
\hline \multirow{4}{*}{$\begin{array}{l}\text { D1: Seguridad } \\
\text { afectiva }\end{array}$} & Experimental & Pre test & 32 & 0 & 5 & 2,53 & 1,135 \\
\hline & & Pos test & 32 & 3 & 6 & 5,16 & ,677 \\
\hline & Control & Pre test & 32 & 1 & 4 & 2,75 & ,950 \\
\hline & & Pos test & 32 & 1 & 5 & 2,50 & 1,164 \\
\hline \multirow{4}{*}{$\begin{array}{l}\text { D2: Libertad de } \\
\text { movimiento }\end{array}$} & Experimental & Pre test & 32 & 1 & 6 & 3,69 & 1,256 \\
\hline & & Pos test & 32 & 3 & 8 & 6,50 & 1,016 \\
\hline & Control & Pre test & 32 & 1 & 7 & 4,06 & 1,458 \\
\hline & & Pos test & 32 & 1 & 8 & 4,25 & 1,814 \\
\hline \multirow{4}{*}{$\begin{array}{l}\text { D3: Espacios y } \\
\text { materiales } \\
\text { apropiados }\end{array}$} & Experimental & Pre test & 32 & 0 & 4 & 2,47 & ,983 \\
\hline & & Pos test & 32 & 4 & 6 & 5,28 &, 523 \\
\hline & Control & Pre test & 32 & 0 & 6 & 2,94 & 1,268 \\
\hline & & Pos test & 32 & 1 & 5 & 2,72 & 1,114 \\
\hline
\end{tabular}

Para el estudio se planteó la hipótesis:

$\mathrm{H}_{0}$ : La aplicación del "Programa para mejorar la autonomía de los niños en etapa pre escolar" no influyó la autonomía, seguridad afectiva, libertad de movimiento, espacios y materiales apropiados de los niños en etapa pre escolar.

H1: La aplicación del "Programa para mejorar la autonomía de los niños en etapa pre escolar" influyó la autonomía, seguridad afectiva, libertad de movimiento, espacios y materiales apropiados de los niños en etapa pre escolar.

Según los datos obtenidos en la tabla 2 se evidenció que el nivel de significancia calculado fue mayor a 0.05 en el pre test, lo cual indicaba que los grupos eran semejantes ( $\left.p_{\text {auto }}=.052 ; p_{\mathrm{d} 1}=.386 ; p_{\mathrm{d} 2}=.290 ; p_{\mathrm{d} 3}=.077\right)$. Luego de la aplicación del programa en el grupo 
experimental el nivel de significancia calculado fue menor a 0.05 , lo que indica que los grupos son diferentes ( $\left.p_{\text {auto }}=.000 ; p_{\mathrm{d} 1}=.000 ; p_{\mathrm{d} 2}=.000 ; p_{\mathrm{d} 3}=.000\right)$, es decir, se rechazó la $\mathrm{H}_{0}$, demostrando que: La aplicación del Programa influyó en la autonomía, seguridad afectiva, libertad de movimiento, espacios y materiales apropiados de los niños en etapa pre escolar, razón por la cual se debería replicar la aplicación del mismo en otras instituciones educativas y así desarrollar progresivamente la autonomía de los niños, que resulta importante para su desenvolvimiento actual y futuro.

\section{Tabla 2}

Prueba de U Mann Whitney para contrastar las hipótesis del estudio

\begin{tabular}{llrrrr}
\hline Prueba & \multicolumn{1}{c}{$\begin{array}{c}\text { Vd: } \\
\text { Autonomía }\end{array}$} & $\begin{array}{c}\text { D1: } \\
\text { Seguridad } \\
\text { afectiva }\end{array}$ & $\begin{array}{c}\text { D2: Libertad } \\
\text { de } \\
\text { movimiento }\end{array}$ & $\begin{array}{c}\text { D3: Espacios } \\
\text { y materiales } \\
\text { apropiados }\end{array}$ \\
\hline Pre test & U de Mann-Whitney & 369,000 & 450,000 & 435,500 & 385,500 \\
& W de Wilcoxon & 897,000 & 978,000 & 963,500 & 913,500 \\
& Z & $-1,942$ &,- 867 & $-1,059$ & $-1,766$ \\
& Sig. &, 052 &, 386 &, 290 &, 077 \\
asintótica(bilateral) & 13,500 & 38,500 & 157,000 & 15,500 \\
Pos test & U de Mann-Whitney & 541,500 & 566,500 & 685,000 & 543,500 \\
& W de Wilcoxon & $-6,725$ & $-6,533$ & $-4,878$ & $-6,851$ \\
& Z &, 000 &, 000 &, 000 &, 000 \\
& Sig. & & & & \\
\hline
\end{tabular}

\section{Discusión}

Para mejorar la autonomía de los niños se requiere siempre del apoyo de los padres de familia o el adulto cuidador, porque es el responsable de la formación del menor, pero a la vez asume con responsabilidad la generación y cumplimiento de las reglas, para no confundir autonomía con libertinaje (Blanco, Umayahara y Reveco, 2004). En este sentido el programa “Aplicación del programa para mejorar la autonomía de los niños en etapa pre escolar” se aplicó en las casas de los menores, en el lugar donde se desenvuelven diariamente, porque por el estado de emergencia sanitario declarado por el gobierno peruano, las escuelas fueron cerradas hasta próximo aviso, sin embargo la educación y formación de los menores no puede afectarse, por ello se implementó para la educación básica regular el programa "Aprendo en casa", el cual se transmite por los canales de televisión, pero el equipo de maestros donde se realizó el estudio 
en mérito a los resultados de la evaluación de la autonomía en el mes de abril, consideró oportuno realizar un programa e implementarlo virtualmente, por lo tanto, se procedió a conversar con los padres de los niños que tenían menores puntajes, con el objetivo que acepten participar en la investigación, efectivamente el resultado fue positivo, quedando finalmente constituido dos grupos (experimental y control).

Cabe precisar que el desarrollo de los niños es mejor cuando se realiza en su entorno natural, porque es allí donde se siente cómodo, seguro y está rodeado de su familia (Barrable, 2019). En este sentido, es meritorio reconocer el apoyo, así como la responsabilidad de la madre, porque la mayoría de ellas participó en todo el proceso de aplicación del programa, asumieron a la vez el papel de la maestra porque explicaban y disponían de los recursos materiales para que los niños realicen las actividades, a la vez asumieron actitudes positivas como motivar y premiar al niño cuando lograba realizar las actividades, aprendieron a comer solos y hacer el uso correcto de los cubiertos (Arciniegas, y otros, 2018; Ministerio de Educación, 2013).

En este sentido, el desarrollo de la autonomía fue un proceso sinérgico entre padres y maestros, donde ha primado el cambio de hábitos de los padres de familia, mejoró las relaciones humanas entre padres e hijos, asumieron con mayor responsabilidad el cuidado y desarrollo del menor, pero, sobre todo, los lazos familiares se fortalecieron y el niño mejoró su autonomía (Gómez y Martin, 2013).

Los resultados coinciden con Ayllón et al. (2019) porque el desarrollo de la autonomía se logra cuando el niño realiza actividades cotidianas, como fueron asearse, comer, cambiarse de ropa, guardar sus juguetes, entre otros. También fue importante el juego con reglas y libre desarrollado en las sesiones de clase, a pesar que al inicio el menor quería desarrollar las actividades como mejor consideraba, en este sentido los ambientes de la casa como sala o comedor se convirtieron en los espacios idóneos para el experimento, por ser amplios y el lugar donde permanecen el mayor tiempo, también fue importante el dormitorio en concordancia con lo planteado por Borgogni (2019) porqué aseveró que la autonomía se logra a través del juego.

También para el estudio se consideró la experiencia de Arizaca (2010), porque los padres en su mayoría sobreprotegen a los menores en los primeros años de vida, razón por la cual el programa estaba dirigido a los niños, pero en esta oportunidad involucró también a los padres, quienes semanalmente recibían una charla sobre las actividades a realizarse en la semana próxima, los objetivos y materiales que se necesitarían, así como el papel que asumirían. Al mismo tiempo, los resultados armonizan con Condori y Luque (2019) porque a 
través del programa los menores lograron el hábito de lavarse las manos, los dientes, bañarse y respetar a los mayores.

Lo antes expuesto, se logró porque los maestros desplegaron sus capacidades pedagógicas antes durante y después de cada sesión de aprendizaje, coincidiendo con lo realizado por Yábar y Bronzoni (2018). Por otra parte, la intervención se sustentó en la teoría de Piaget, porque el niño debe adquirir nuevos conocimientos y hábitos según la edad cronológica (Arias, Merino, y Peralvo, 2017), también se el programa se estructuró en función a la teoría sociocultural de Vigotsky porque las actividades se desarrollaron en el lugar donde se desenvuelve diariamente, al mismo tiempo se fundamentó en el aprendizaje significativo de Ausbel porque el menor aprendió aquellas actividades que para el resultaban ser significativas (Chaves, 2001; De Rosa, 2018; Baca, 2018; Carrera y Mazzarella, 2001).

Los hallazgos encontrados exponen la situación de la autonomía de los niños en etapa pre escolar según la población de estudio, en este sentido es importante que para las futuras investigaciones se incremente el número de estudiantes y además se diseñe previamente un programa para los padres de familia, de tal forma que se logre resultados trascendentales.

Las limitaciones que se tuvieron estuvieron alrededor de la disponibilidad del material concreto para las actividades sincrónicas, asimismo el problema de conexión con internet fue reiterativo, el mismo que obligaba a suspender la sesión y reprogramar para una próxima fecha, además ello implicaba que los padres de familia reorganicen sus actividades para que participen en la experiencia de aprendizaje, por otra parte, hubieron niños que no disponían de Pc con cámara y audio, razón por la cual utilizaban el celular, además resultó incómodo y desagradable, porque la clase se interrumpía por una llamada, los cuáles se han superado progresivamente, lo cual no interfirió en los resultados.

En este caso, los futuros estudios podrían desarrollar una investigación cualitativa, donde a partir de la observación identifiquen problemas de los infantes, luego diseñar un nuevo programa de intervención de acuerdo a la necesidad y en concordancia con el logro de los objetivos institucionales.

\section{Conclusión}

La autonomía es un tema importante en el Currículo Nacional de la educación Básica Regular, el mismo que debe ser practicado en el nido y reforzado en el seno familiar, sin embargo ante la situación que se vive en el presente año, lamentablemente en el mes de marzo los niños no recibieron el aprestamiento debido, y cuando se realizó la evaluación con la lista de chequeo lamentablemente los menores carecían de autonomía, por ello es que se implementó 
el programa con una duración de 12 semanas, evidenciándose una mejora notable en el grupo experimental, respecto al grupo control, a pesar que al inicio se encontraba en mejor situación.

Por lo tanto, a la luz de los resultados es importante y urgente que se implementen programas de intervención al respecto en la institución con el grupo control, de tal forma que al finalizar el periodo escolar no se evidencia diferencias entre los grupos.

\section{Referencias}

Arciniegas, M., Puenres, A., Trujillo, E., Gamba, M., Gutierréz, C., \& Beltrán, A. (2018). Estilos parentales, función familiar y rendimiento académico: un estudio de caso de seis familias de un colegio público en Bogotá, Colombia. Voces y Silencios: Revista Latinoamericana de Educación, 9(2), 4-36. Obtenido de https://revistas.uniandes.edu.co/doi/pdf/10.18175/vys9.2.2018.02

Arias, W. (2015). Jerome Bruner 100 años dedicados a la psicología la educación y la cultura. Revista peruana de Historia de la Psicología, 1, 59-79. Obtenido de https://www.researchgate.net/publication/299358301_Jerome_Bruner_100_anos_dedi cados_a_la_psicologia_la_educacion_y_la_cultura

Arias-Arroyo, P., Merino-Zurita, M., \& Peralvo-Arequipa, C. (2017). Análisis de la Teoría de Psico-genética de Jean Piaget: Un aporte a la discusión. Dom. Cien., 3(3), 833-845. doi:10.23857/dom.cien.pocaip.2017.3.3.jun. 833-845

Arizaca, L. (2019). El grado de relación de la sobreprotección de los padres de familia en la autonomía del niño y niña de 4 años de la institución educativa inicial No285 Gran Unidad Escolar San Carlos de la ciudad de Puno - 2018. (Tesis de licenciatura). Puno: Unviersidad Nacional del Altiplano. Obtenido de http://repositorio.unap.edu.pe/handle/UNAP/11979

Ayllón, E., Moyano, N., Lozano, A., \& Cava, M. (2019). Parents'Willingness and Perception of Children's Autonomy as Predictors of Greater Independent Mobility to School. International Journal of Environmental Research and Public Health, 16, 1-14. doi:10.3390/ijerph16050732

Baca, C. (2018). Contextos de producción de la teoría de Lev Vigotsky, a 120 años de su nacimiento. Córdoba: Universidad Nacional de Córdoba. Obtenido de https://pdfs.semanticscholar.org/7494/a7e5d0197dea8327b49e77c1b9a74aac68c3.pdf 
Barrable, A. (2020). Shaping space and practice to support autonomy: lessons from natural settings in Scotland. Learning Environments Research, 23, 291-305. doi:10.1007/s10984-019-09305-x

Blanco, R., Umayahara, M., \& Reveco, O. (2004). Participación de las familias en la educación infantil latinoamericana. Santiago de Chile: UNESCO. Obtenido de https://www.oei.es/historico/inicial/articulos/participacion_familias.pdf

Borgogni, A. (2019). Gli spazi pubblici come luoghi educativi: autonomia, mobilità indipendente e stili di vita attivi nei bambini. Pedagogia Oggi, 7(1). doi:10.7346/PO012019-19

Carrera, B., \& Mazzarella, C. (2001). Vygotsky: enfoque sociocultural. Educere. La Revista Venezolana de Educación, 5(13), 41-44. Obtenido de https://www.redalyc.org/pdf/356/35601309.pdf

Chaves, A. (2001). Implicaciones educativas de la teoría sociocultural de Vigotsky. Revista Educación, 25(2), 59-65. Obtenido de https://www.redalyc.org/pdf/440/44025206.pdf

Cuervo, A. (2010). Pautas de crianza y desarrollo socioafectivo en la infancia. Diversitas. Perspectivas en psicología, 6(1), 111-121. Obtenido de https://www.redalyc.org/pdf/679/67916261009.pdf

De Rosa, P. (2018). Enfoque psicoeducativo de Vigotsky y su relación con el interaccionismo simbólico: Aplicación a los procesos educativos y de responsabilidad penal juvenil. Propósitos y Representaciones, 6(2), 631-669. doi:10.20511/pyr2018.v6n2.246

Gómez, O., \& Martin, J. (2013). Cómo fomentar la autonomía y responsabilidad en nuestros hijos e hijas (Vol. 1). Madrid: IO Sistemas de Comunicación. Obtenido de http://cppomove.educa.aragon.es/IMG/pdf/-24.pdf

Hernández, R., Fernández, C., \& Baptista, M. (2014). Metodología de la investigación. México: McGraw-Hill.

Martakis, K., Brand, H., \& Schroder-Back, P. (2018). Desarrollo de la autonomía del niño en la atención pediátrica: hacia un modelo ético. Arch. Argent. Pediatr., 116(3), 401-408. doi:.5546/aap.2018.e401 
Martin-Romera, A., \& Molina, E. (2017). Valor del conocimiento pedagógico para la docencia en Educación Secundaria: Diseño y validación de un cuestionario. Estudios Pedagógicos, XLIII(2), 195-220. doi:10.4067/S0718-07052017000200011

Ministerio de Educación. (2013). El valor educativo de los cuidados infantiles para la atención de los niños y niñas de 0 a 3 años. Lima: MINEDU. Obtenido de http://www.minedu.gob.pe/pdf/ed/guia-de-cuidados-infantiles.pdf

Ministerio de la mujer y Poblaciones Vulnerables. (2012). Plan Nacional de Acción por la Infancia y la adolescencia 2012 - 2021. Lima: MMPV. Obtenido de https://www.mimp.gob.pe/webs/mimp/pnaia/pdf/Documento_PNAIA.pdf

Morrison, G. (2005). Educación infantil. Madrid: Pearson Educacion.

Oliveira, D. (2019). Autonomia dos bebês e das crianças pequenasocência na creche: a modulação da presença dos adultos na construção da. Poiésis - Revista do programa de pós-graduação em educação - Mestrado - Universidade Do Sul de Santa Catarina, 13(24), 240-255. doi:10.19177/prppge.v13e242019240-255

Peñaranda, Y. (2018). Niños y niñas en proceso de desarrollo de la autonomía en la IEI $N^{o} 208$ Barrio Laykakota. (Tesis de segunda especialidad). Lima: Universidad San Ignacio de Loyola. Obtenido de http://repositorio.usil.edu.pe/handle/USIL/6692

Posada, Á., Gómez, J., \& Ramírez, H. (2005). El niño sano. Bogotá: Editorial Médica Internacional.

Rivas-Ruiz, R., Moreno-Palacios, J., \& Talavera, J. (2013). Investigación clínica XVI Diferencias de medianas con la U de Mann-Whitney. Revista Médica del Instituto Mexicano del Seguro social, 51(4), 414-419. Obtenido de https://www.medigraphic.com/cgi-bin/new/resumen.cgi?IDARTICULO=43670

Salazar, L. (2017). Autonomía personal y salud infantil. Málaga: IC Editorial.

Vygotsky, L. S. (1978). Mind in Society: The Development of Higher Psychological Processes . Edited and Translated by M.Cole, V.John-Steiner, S.Scribner, and E.Souberman . USA: Harvard University Press.

Yábar, C., \& Bronzoni, L. (2018). desarrollo de la autonomía en niños de 18 a 24 meses de edad según la filosofía de Reggio Emilia aplicada en el Nido La Casa Amarrilla. (Tesis 
de licenciatura). Lima: PUCP. Obtenido de http://tesis.pucp.edu.pe/repositorio/handle/20.500.12404/12799

Ziaei, S., Contreras, M., Zelaya, E., Persson, L., Hjerm, A., \& Ekstrom, E. (2015). Women's autonomy and social support and their associations with infant and young child feeding and nutritional status: community-based survey in rural Nicaragua. Public Health Nutr., 18(11), 1979-1990. doi:10.1017/S1368980014002468 